\title{
El pie diabético
}

El pie diabético es una complicación grave de la diabetes con mal control metabólico habitual, caracterizada fisiopatológica y clinicamente por la confluencia de la neuropatía diabética periférica con la macrovasculopatía y la microangiopatía y asociadas con frecuencia a alteraciones mecánicas del pie junto con mayor facilidad para el desarrollo de infecciones. Su etiología es multifactorial, pudiendo predominar en unos casos la lesión vascular y en otros la neurológica, si bien lo más común es que el paciente presente una lesión mixta en la que existen una serie de factores predisponentes (edad, mal control metabólico, vasculopatía y neuropatía), factores precipitantes (calor, traumatismos, etc.) y factores agravantes como la isquemia aguda, las úlceras neuropáticas y la infección local (1).

A su vez el propio pie diabético puede ser considerado un marcador pronóstico de dicha enfermedad, observándose que la supervivencia entre los 3 y los 5 años posteriores a la amputación sólo alcanza el 40-50\% mientras que el 50\% de los pacientes amputados de una extremidad presentan complicaciones en el pie contralateral al cabo de 2 años, existiendo otro $50 \%$ de posibilidad de nueva amputación entre los 3 y los 5 años siguientes.

En el pie diabético no complicado, puede diferenciarse un síndrome angiopático y un síndrome neuropático. El síndrome angiopático provocado por la isquemia crónica se inicia con la aparición de claudicación intermitente seguido por dolor en reposo así como frialdad y palidez progresivas. Se debe realizar la toma de pulsos arteriales desde femoral a la pedia, pero hoy en día es casi imprescindible la realización del estudio eco-Doppler previo a la realización de arteriografia precoz, que va a ser decisiva de cara al tratamiento.

El síndrome neuropático cursa con parestesias y disestesias acompañadas de dolor, sobre todo nocturno, de extremidades inferiores y suele presentarse antes que el angiopático. En esta fase ya se encuentra pérdida de la sensibilidad térmica y vibratoria así como disminución o abolición de los reflejos aquíleos o rotulianos. El pie diabético complicado se caracteriza por la aparición de ulceración, infección o gangrena. La úlcera neuropática o mal perforante plantar es la lesión más característica del pie diabético complicado, agravándose el pronóstico en el curso evolutivo del pie. La infección es fácil y frecuente, pudiéndose llegar a producir osteomielitis de los huesos del pie afecto. La palpación minuciosa y la radiografia nos ayudan a establecer un diagnóstico precoz de la infección por anaerobios. La gangrena es el estadio final de la isquemia aguda o crónica y constituye una alteración que empeora considerablemente el pronóstico del pie y del paciente. El dolor en la isquemia es variable, pero generalmente intenso. En el trabajo de Real Collado y cols. (2) se han estudiado los factores asociados con amputación en 14 diabéticos $(9,2 \%)$ de un grupo de 152, que acudieron a la Unidad del Pie Diabético con ulceraciones en los pies, en un periodo de 2,5 años. Los autores señalan como factores asociados la amputación previa (riesgo incrementado 3,7 veces), la retinopatía proliferativa y la osteomielitis, así como de forma independiente la vasculopatía en miembros inferiores (riesgo aumentado 7,1 veces) y la infección grave (riesgo aumentado 14,4 veces). En España, según Calle Pascual y cols. (3) la incidencia de amputaciones no traumáticas es casi 30 veces mayor entre los diabéticos respecto a la población no diabética, mientras que en el Reino Unido esa diferencia es sólo 2,4 veces mayor (4), aunque en el conjunto de la población sea casi 6 veces mayor, quizá por una mayor prevalencia de aterosclerosis en la población general respecto a la población mediterránea.

En todo caso la necesidad de amputación en el 9,2\% de los diabéticos que acudieron a la Unidad en 2,5 años, es suficientemente importante para recomendar cambios en la sociedad en que vivimos, motivados por su impacto en la persona afectada, en su familia y las consecuencias sociales y laborales, además de las económicas. De siempre se ha dicho que la prevención es menos costosa que la enfermedad, en este caso la amputación. Sin embargo la política sanitaria en muchas de las comunidades autónomas aún no es consciente del problema, o le da de lado para evitar la inversión inicial que siempre es necesaria. Por un lado, hace falta incrementar las medidas de educación sanitaria (Educación Diabetológica), no sólo entre los diabéticos y sus familiares, sino también entre los médicos generales (Médicos de Familia) y el personal sanitario de enfermería, de forma que sean valoradas adecuadamente las úlceras de el pie del diabético y sean tratadas con cono- 
cimiento y entusiasmo hasta su curación, muchas veces solo con tratamiento conservador (5) evitando amputaciones o reduciéndolas al mínimo. Por otra parte, hace falta dotar de medios a los Servicios de Endocrinología, facilitando la creación y funcionamiento de Secciones de Diabetes, con su Unidad de Educación Diabetológica, su Hospital de Día y su Unidad Multidisciplinaria del Pie Diabético capaz de coordinar los conocimientos diversos para una adecuada valoración de los factores implicados (6) junto a la aplicación de las medidas más modernas en su profilaxis y tratamiento (7). Crear un Consejo Asesor de Diabetes para "salir en la foto" y poco más, es totalmente insuficiente. Hace falta seguir las recomendaciones de los técnicos en la materia, en este caso los diabe-

\section{Bibliografía}

1. Edmonds M and Foster AVM. Diabetic foot. En "Diabetic Complications" de KM Shaw. Ed: Wiley \& Sons Chichester UK 1996; 7: 149-178.

2. Real Collado JT, Valls M, Basanta Alario ML, Ampudia Blasco FJ, Ascaso Gimilio JF y Carmena Rodríguez R: Estudio de factores asociados con amputación en pacientes diabéticos con ulceración en pie. An Med Interna (Madrid) 2001; 18: 59-62.

3. Calle-Pascual AL, Redondo MJ, Ballesteros M, Martínez-Salinas MA Díaz JA, De Matías P, et al. Nontraumatic lower extremity amputations in diabetic and non diabetic subjects in Madrid, Spain. Diabetes \& Metabolism Paris 1997; 23: 519-523.

4. Morris AD, Mc Alpine R, Syeinke D, Boyle DL, Ebrahim AR, Vasudev $\mathrm{N}$, et al. Diabetes and lower limb amputation in a community. A retros- tólogos (endocrinólogos). De esa forma la política sanitaria (diabetológica) será no sólo menos costosa en lo económico (8), sino también más rentable social y laboralmente, consiguiendo, además, un incremento de la calidad de vida del diabético, que en definitiva es lo que humanamente cuenta.

A. JARA ALBARRÁN

Servicio de Endocrinología. Hospital General Universitario Gregorio Marañón. Madrid pective cohort study DARTS/MEMO collaboration. Diabetes audit and research in Tayside Scotland/Medicines monitoring unit. Diabetes Care 1998; 21738-43.

5. Pittet D, Wyssa B, Herter-Clavel C, et al. A retrospective cohortt study with long-term follow-up. Arch Intern Med 1999; 159: 851-6.

6. Caputo GM, Cavanagh PR, Ulbrech JS, Gibbons GW and Karchmer AW. Assessment and management of foot disease in patients with diabetes. N Engl J Med 1994; 331: 854-60.

7. Edmonds ME. Progress in care ofthe diabetic foot. Lancet 1999; 354 270-72.

8. Harrington $\mathrm{C}$, Zagari MJ, Corea $\mathrm{J}$ and Klitenic $\mathrm{J}$. A cost analysis of diabetic lowerextremity ulcers. Diabetes Care 2000; 23: 1333-38. 